\title{
Injury prevention in football: Knowledge and behaviour of players and availability of medical care in a Nigerian youth football league
}

\author{
O B A Owoeye, ${ }^{1}$ BPT, MSc; S R A Akinbo, $,{ }^{1} \mathrm{BSc}, \mathrm{MSc}, \mathrm{PhD} ;$ O A Olawale, ${ }^{1} \mathrm{BSc}, \mathrm{MSc}, \mathrm{PhD} ; \mathbf{B}$ A Tella, ${ }^{1} \mathrm{BSc}, \mathrm{MSc}, \mathrm{PhD}$ \\ N M Ibeabuchi, ${ }^{2} \mathrm{MBBS}, \mathrm{MSc}, \mathrm{PhD}$ \\ ${ }^{1}$ Department of Physiotherapy, Faculty of Clinical Sciences, College of Medicine, University of Lagos, Nigeria \\ ${ }^{2}$ Department of Anatomy, Faculty of Basic Medical Sciences, College of Medicine, University of Lagos, Nigeria
}

Corresponding author: O B A Owoeye (obowoeye@unilag.edu.ng)

Background. Exposure to competitive football is increasing among male youth football players in Nigeria. However, medical support to abate the impact of injuries appears inadequate and there is limited literature to show whether youth football players are knowledgeable about, and practise effective measures for injury prevention in football (IPF).

Objective. To assess the knowledge and behaviour of male youth football players regarding IPF and the availability of medical care for players.

Methods. We conducted a cross-sectional study among all registered first-division players of a male youth football league in Lagos, Nigeria. Using a self-administered questionnaire, we assessed players' knowledge regarding IPF, awareness of the Fédération Internationale de Football Association (FIFA) 11+ injury-prevention programme, injury-prevention behaviour and availability of medical attendants during training and competitive matches.

Results. The mean age of the players was 18.5 years (standard deviation (SD) \pm 1.7 ; range 12 - 19). Their overall mean knowledge score regarding IPF was 4.40 ( $\mathrm{SD} \pm 1.92$ ) from a total score of 9 , with the majority falling into the poor $(39.1 \%)$ and fair $(43.9 \%)$ knowledge categories. Most (79.3\%) players were not aware of the FIFA 11+ programme. Less than half (40.5\%) wore shin guards during training sessions, while $52.5 \%$ reported wearing shin guards during matches. Less than two-thirds always warmed up or cooled down at training or matches. About three-quarters $(73.1 \%)$ and over half (52.1\%) reported not having medical attendants working with their teams during matches and training, respectively.

Conclusion. There is a clear deficiency in the knowledge and behaviour of injury-prevention measures among Nigerian male youth football players, and adequate medical care is lacking. There is a need for injury-prevention advocacy and implementation of effective interventions to bridge the identified deficiencies in youth football in Nigeria.

S Afr J SM 2013;25(3):77-80. DOI:10.7196/SAJSM.471

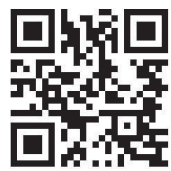

Football (soccer) players are known to suffer relatively high rates of injury compared with participants in other sports, ${ }^{[1,2]}$ and youth football players are no exception to this. ${ }^{[3]}$ Young people are particularly at risk of sports injury because of high levels of exposure at a time of major physiological change. ${ }^{[4]}$ The importance of injury prevention in football (IPF) among young players cannot be overemphasised as talented players need to stay injury-free as far as possible, to get to the peak of their careers. Implementation of injury-prevention measures in this extremely vulnerable population of disadvantaged youth is therefore imperative.

A set of programmes such as core stability, proprioception and strength training, dynamic stretching, protective and suitable equipment, appropriate surface as well as appropriate training, adequate recovery, psychology and nutrition have been described as main components of injury prevention and rehabilitation in football. ${ }^{[2,4-8]}$

Football players in Nigeria are faced with huge challenges. A major problem is the low level of financial resources for professional, amateur and youth clubs, most of which are not well funded, especially those owned by the government. ${ }^{[9]}$ Appropriate facilities, personnel and equipment are usually lacking and this is even worse at the youth football level. Consequently, players are faced with factors that predispose them to injuries that militate against their football career.

It has being emphasised that the prevention of injuries should always be a priority and is even more important when treatment possibilities are restricted, as in many parts of Africa. ${ }^{[10]}$ To enable the implementation of prevention programmes, knowledge of preventive measures among sports participants is imperative. Regulatory bodies and team owners are obliged to provide adequate information and training on injury-prevention strategies to their players. ${ }^{[11]}$ In view of this, the Federation of International Football Associations (FIFA) through the FIFA Medical Assessment and Research Centre (F-MARC) has developed a neuromuscular injury-prevention programme, referred to as FIFA 11+, to help reduce the incidence of injuries in football. ${ }^{[12]}$

FIFA $11+$ is a complete warm-up programme developed by F-MARC and experts in the field of IPF to reduce injuries among male and female football players aged $\geq 14$ years. ${ }^{[13]}$ However, the 
level of awareness and implementation of the FIFA $11+$ among footballers, coaches and sports medicine personnel in Nigeria is not known. Furthermore, there is generally limited evidence to show whether or not youth football players are knowledgeable about effective measures for IPF and whether they actually practise such.

To date, no study has investigated the knowledge and behaviour relating to IPF in male youth football. The aim of this study was therefore to assess the knowledge and behaviour of male youth football players regarding IPF and the availability of medical care for players.

\section{Methods}

A cross-sectional cohort study was conducted on all registered first-division team players of a male youth football league in Lagos, Nigeria (Lagos Junior League). All first-division players of the 14 teams (a total of 260 players) registered for the 2011/2012 league season were invited to participate in the study.

A self-administered questionnaire, adapted from previous studies ${ }^{[11,14]}$ and modified to address the specific objectives of the study, was used for data collection. The questionnaire was pilot-tested among 16 players of a second-division team of the Lagos Junior League. Players were asked to report any ambiguities in questions or the format of the questionnaire. Minor revisions were made on the basis of feedback from the pilot study. For the purpose of comprehension among players, 'water consumption' was used as a synonym for fluid replacement or rehydration in the questionnaire. The questionnaire comprised three sections: section A assessed players' demographics; section $B$ assessed players' knowledge regarding IPF and awareness of the FIFA 11+ injury-prevention programme; and section $C$ assessed players' injuryprevention behaviour and the availability of medical personnel for competitive matches and training sessions.

The questionnaire was distributed to all players towards the end of the 2011/2012 league season. The study was carried out between February and March, 2012. The various teams were visited consecutively. Questionnaires were self-administered on training grounds after the delivery of brief information on the objectives of the study and instructions on how to complete the questionnaire. A questionnaire was considered to be invalid for data entry if: (i) less than $50 \%$ of the returned questionnaire was completed (based on the total number of questions); or (ii) the questionnaire was returned to the researcher or research assistant later than the time of administration.

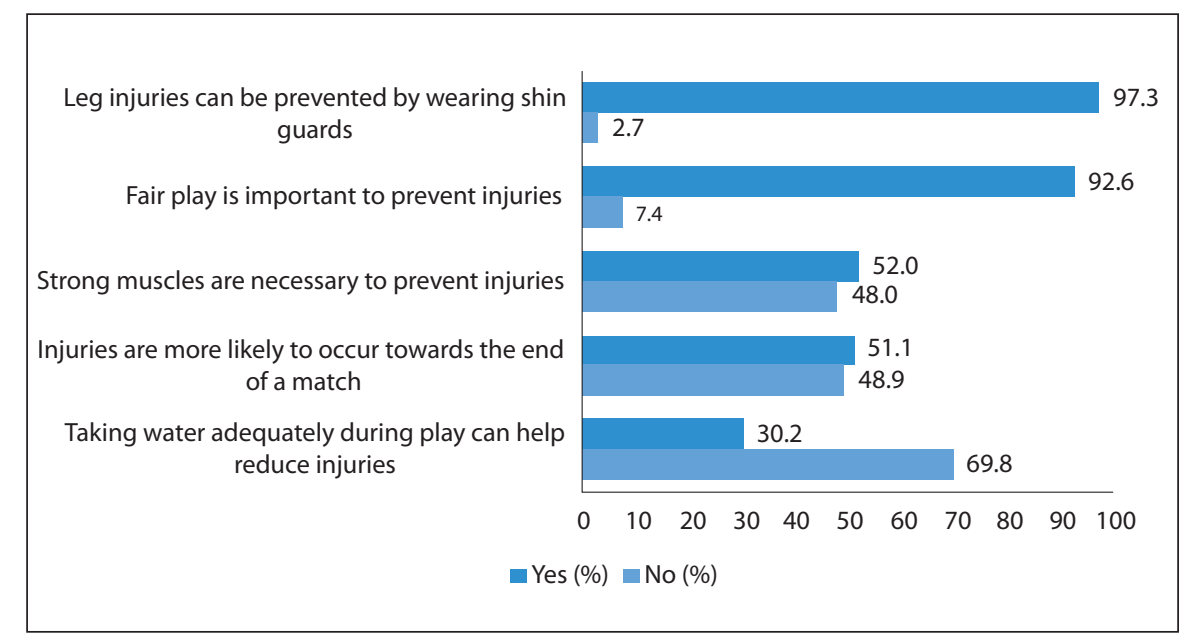

Fig. 1. Players' knowledge on injury prevention in football.

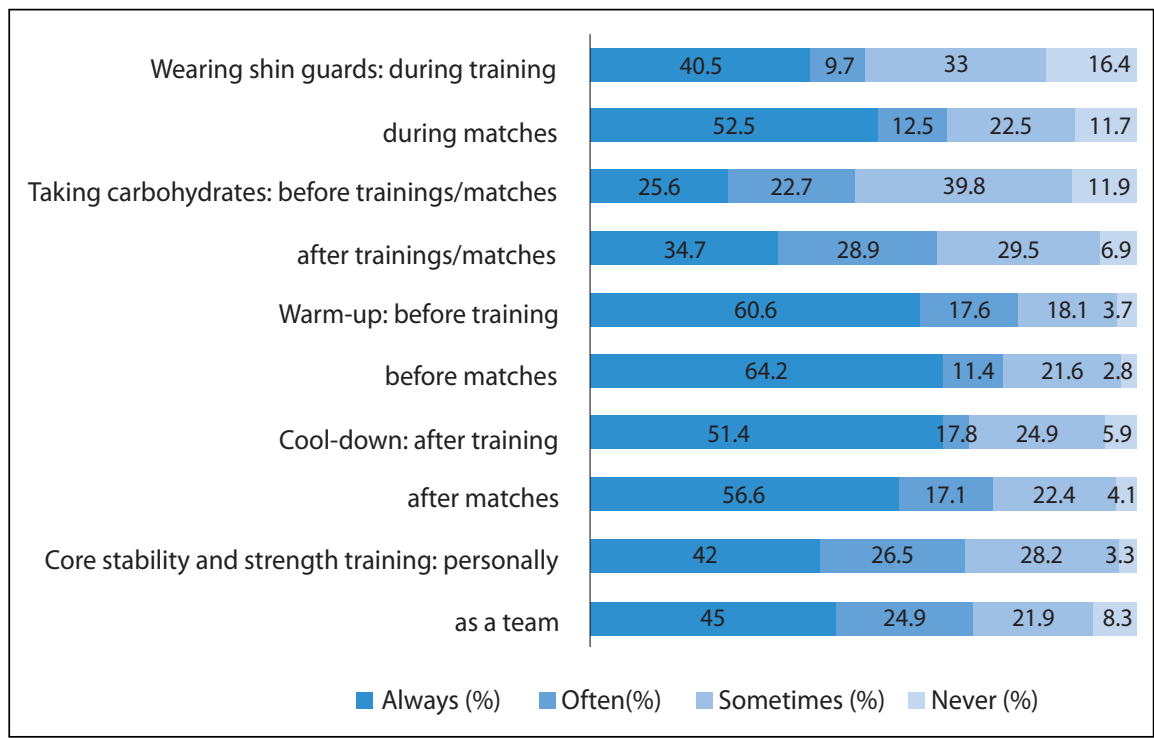

Fig. 2. Injury-prevention behaviour of players.

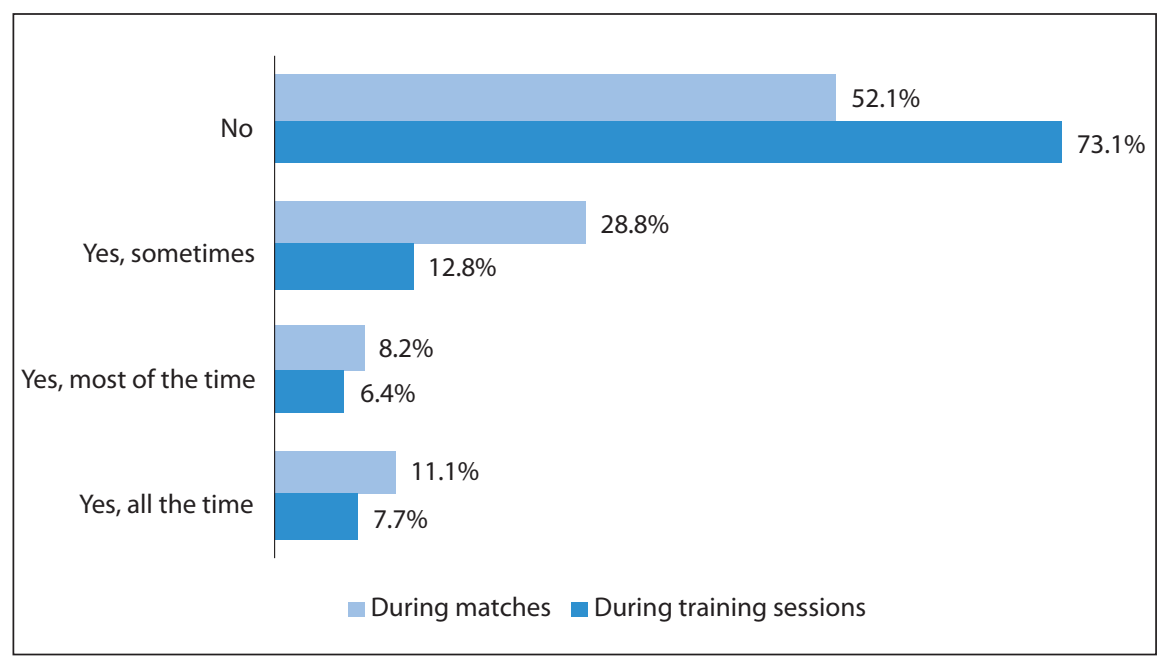

Fig. 3. Availability of medical attendants during matches and training sessions. 
Data were summarised using frequencies, percentages and means ( \pm standard deviations (SDs)) and cross-tabulation chi-square analyses were done as required. A 9-point knowledge scale for the assessment of players' knowledge regarding IPF was generated from the questionnaire. Based on the knowledge score range, players' overall knowledge was categorised as poor (0 - 3 points), fair (4 - 6) or good (7 - 9). Statistical significance was set at $p<0.05$.

Ethical approval of the study was obtained prior to its commencement. Approval to conduct the study was also obtained from the league administrators for official access to the various football teams included in the research.

\section{Results}

\section{Player demographics and IPF knowledge}

A total of 212 of the 260 questionnaires distributed were returned (81.5\% response rate); however, only 182 were valid for data analysis. The players' mean body mass index (BMI) was $21.95 \mathrm{~kg} / \mathrm{m}^{2}(\mathrm{SD} \pm 4.39)$ and mean age was 18.5 years ( $S D \pm 1.7$; range 12 - 19) with 9.75 years (SD \pm 3.71 ) of football experience. In terms of player position, $14 \%$ were goalkeepers, while $31.2 \%, 30.6 \%$ and $24.2 \%$ were defenders, midfielders and strikers, respectively.

Based on the 9-point IPF knowledge scale, the overall mean knowledge score was 4.40 ( $\mathrm{SD} \pm 1.92$ ). Based on the knowledge score range, most players fell within the poor (score 0 - 3) and fair (score 4 - 6) knowledge categories ( $39.1 \%$ and $43.9 \%$, respectively).

Fig. 1 presents specific questions regarding players' knowledge of IPF. The majority of the players correctly identified that wearing shin guards and fair play behaviour were important for IPF $(97.3 \%$ and $92.6 \%$, respectively). However, a large proportion of the players (69.8\%) wrongly indicated that adequate consumption of water (rehydration) was not important for IPF and nearly half (48.0\%) also wrongly indicated that strong muscles were not important for IPF.

Over two-thirds (69.7\%) of players claimed to be knowledgeable about IPF. However, $90.6 \%$ wished to know more about IPF. The majority (79.3\%) of the players had never heard about the FIFA 11+ warm-up programme before the time of the study.

\section{Football experience, playing position and IPF knowledge}

Table 1 shows the relationship between players' years of football experience, playing position and knowledge of IPF. The players' mean knowledge score decreased slightly with increasing years of football experience. However, no statistically significant relationship was found between years of football experience and players' knowledge of IPF ( $p=0.737$ ). Also, no significant relationship was found between players' positions and knowledge scores $(p=0.732)$.

\section{Injury-prevention behaviour of players}

Fig. 2 shows the injury-prevention behaviour of players. Less than half $(40.5 \%)$ of the players reported always using shin guards during training sessions and only $52.5 \%$ reported always wearing shin guards during matches. About one-sixth (16.4\%) and $11.7 \%$ of players never used shin guards during training and matches, respectively. A few of the players consciously consumed food or snacks rich in carbohydrates before (25.6\%) and after (34.7\%) play. Less than twothirds of players always warmed up or cooled down at training or matches.
Table 1. Relationship between players' knowledge, football experience and playing position

\begin{tabular}{lllll}
\hline & & \multicolumn{3}{c}{ Knowledge score } \\
\cline { 3 - 5 } Variable & $\boldsymbol{n}$ & mean $( \pm \mathrm{SD})$ & $\boldsymbol{F}$-value & p-value \\
\hline Football experience & & & 0.31 & 0.737 \\
$\quad$ <5 years & 31 & $4.61( \pm 1.82)$ & & \\
$\quad$ 6 - 10 years & 78 & $4.46( \pm 2.08)$ & & \\
$>$ 10 years & 73 & $4.30( \pm 1.84)$ & & \\
Playing position & & & 0.432 \\
$\quad$ Goalkeeper & 25 & $4.84( \pm 1.68)$ & & \\
$\quad$ Defender & 56 & $4.41( \pm 1.88)$ & & \\
$\quad$ Midfielder & 54 & $4.33( \pm 2.15)$ & & \\
Striker & 44 & $4.47( \pm 1.80)$ & &
\end{tabular}

Table 2. Self-reported medical attendants persent during matches and training sessions

\begin{tabular}{|c|c|}
\hline Medical attendant ${ }^{*}$ & $\%$ \\
\hline Masseur & 55.8 \\
\hline Physiotherapist & 36.0 \\
\hline Nurse & 32.6 \\
\hline Physician & 29.1 \\
\hline Pharmacist & 11.6 \\
\hline Dentist & 10.5 \\
\hline
\end{tabular}

\section{Availability of medical attendants}

About three-quarters (73.1\%) and over half (52.1\%) of the players did not have medical attendants working with their teams during training sessions and matches, respectively (Fig. 3). For the few players that seldom, often or always had medical attendants, the masseur (55.8\%) and the physiotherapist (36.0\%) were mostly reported as available (Table 2).

\section{Discussion}

Risk communication has been argued to be the most essential element of a risk-management process, because without effective communication strategies, risk mitigation will not be accessible to stakeholders. ${ }^{[8]}$ This study found that only a few players in the study population had a good knowledge of IPF and the overall mean knowledge score was below average. Furthermore, the majority of the players had never heard about the FIFA 11+ injury-prevention programme and its implementation among football players. This implies that the majority of these players were not well exposed to current information relating to IPF. However, almost all players wished to know more about IPF.

Proprioception, core muscle strength and stability training have been established in the literature as components of IPF ${ }^{[5,6,13]}$ A neuromuscular training programme such as the FIFA $11+$ is one football-specific injury-prevention programme proven to be effective in improving physical performance and reducing injury risk among young female players ${ }^{[13,15]}$ However, implementation of this programme appears to be limited by the low level of awareness among players and coaches in 
the region. The present study reveals that the majority of the players were not aware of the FIFA 11+ programme. Although the programme is yet to be tested in young male players, ${ }^{[15]}$ its implementation, based on present evidence, may help to abate the risk of injury in male youth football considering its potential in improving neuromuscular fitness.

The sequelae of dehydration (mostly distorted body homeostasis, fatigue and impaired physical performance) predispose players to an increased risk of injury and illness in football. ${ }^{[8,16-17]}$ Adequate hydration during matches and training sessions has been advised as an important measure to minimise injury risk, particularly heat cramps and heat illnesses in football, and particularly in hot environments. ${ }^{[7,8,17]}$ Also, fair play has been documented as an established measure for IPF. ${ }^{[8]}$ Although most of the players knew that fair play was necessary to minimise the occurrence of injuries in football, about half of the players did not know that strong muscles and rehydration were important components of IPF. Furthermore, about half of them did not know that the chances of sustaining an injury increases towards the end of a competitive match. ${ }^{[1]}$ The aforementioned form some of the evidence-based information that football players are expected to have in order to avert injuries.

No significant relationship was found between players' football experience, positions and knowledge score concerning IPF. This suggests that the number of years of experience and position of play do not determine how much the young football player knows about injury-prevention strategies in football.

The use of shin guards during football matches and training sessions is essential for lower-leg injury prevention, especially contact injuries to the shin. ${ }^{[5]}$ Also, consumption of carbohydrate-rich food or drinks prior to participation in matches and training sessions has been documented as necessary, and advocated for IPF. ${ }^{[7]}$ However, there were major deficiencies in the injury-prevention behaviour of players. The main deficiencies identified related to the use of shin guards during training and matches, carbohydrate intake before and after training and matches, and cool downs before and after training and matches, respectively.

As part of injury prevention, adequate injury management and rehabilitation are essential; especially in the prevention of re-injury. Unfortunately, youth football is often disadvantaged with inadequate or unavailable sports medicine personnel and treatment, particularly in developing countries. This study empirically confirmed that there is limited medical coverage during training and matches in a cohort of male youth football players in Nigeria. This may be attributed to a lack of funds for youth football at the developmental level. The youth football teams that participated in the study are run by local government councils in Lagos State, Nigeria, and a regular complaint from the coaches is the lack of, or sparse fund allocation to run teams properly. Hence, most teams cannot afford the services of qualified medical personnel. This may explain, in part, why most teams that had medical attendants attached to them mainly had masseurs. It is, however, possible that some of the players mistook masseurs and traditional bone setters as qualified health personnel; players often refer to medical attendants whether qualified or not as physiotherapists, nurses or doctors. Thus, the aforementioned qualified personnel might have been over-reported and unqualified personnel under-reported by the players in our study.

Since it is unlikely that qualified medical personnel will be available at the youth football level due to cost and other militating factors, it is important that masseurs and other unqualified attendants who are the main providers of medical services to youth football players, are trained in sports first aid to ensure proper injury management and to prevent re-injuries.

The findings from this study raise the question of whether the latest injury-prevention measures are best disseminated to youth football players. Scientific evidence to show that an injury-prevention strategy works does not guarantee that it will actually prevent injuries in a realworld context if it is not adopted by players and their coaches. ${ }^{[19]}$ Injuryprevention advocacy among players and coaches is imperative for the implementation of effective strategies in youth football. There is a need for future studies to assess barriers to implementation of such strategies for injury prevention in youth football, and this will help to direct plans for implementation.

\section{Conclusion}

In Nigerian male youth football, there is a clear deficiency in the knowledge and behaviour of players regarding injury-prevention measures; medical care is limited and non-qualified personnel predominantly attend to injured players. Injury-prevention advocacy and effective interventions are needed to bridge identified deficiencies.

\section{References}

1. Hawkins RD, Fuller CW. A prospective epidemiological study of injuries in four English professional football clubs. Br J Sports Med 1999;33:196-203.

2. Kirkendal DT, Junge A, Dvorak J. Prevention of football injuries. Asian J Sports Med 2010;1(2):81-92.

3. Emery C. Risk factors for injury in child and adolescent sport: A systematic review of the literature. Clin J Sport Med 2005;13:256-268.

4. Abernethy L, Bleakley C. Strategies to prevent injury in adolescent sport: A systematic review. Br J Sports Med 2007;41:627-638. [http://dx.doi.org/10.1136/bjsm.2007.035691]

5. Olsen L, Scanlan A, MacKay M, et al. Strategies for prevention of soccer related injuries: A systematic review. Br J Sports Med 2004;38:89-94. [http://dx.doi.org/ 10.1136/ bjsm.2002.003079]

6. Mandelbaum BR, Silvers HJ, Watanabe DS, et al. Effectiveness of a neuromuscular and proprioceptive training program in preventing anterior cruciate ligament injuries in female athletes: 2-year follow-up. Am J Sports Med 2005;33:1003-1010.

7. Consensus Statement: Nutrition for Football: FIFA/F-MARC Consensus Conference. J Sports Sci 2006;24(7):663-664.

8. Fuller CW, Junge A, Dvorak J. Risk management: FIFA's approach for protecting the health of football players. Br J Sports Med 2012;46:11-17. [http://dx.doi.org/10.1136/ bjsports-2011-090634]

9. Agiri A. TAIWO OGUNJOBI Offers Info On How To Revive Nigeria Sports. http://www. allnigeriasoccer.com/read_news.php?nid=7149 (accessed 12 March 2013).

10. Constantinou D. Football injuries - surveillance, incidence and prevention. Continuing Medical Education 2010;28(5):220.

11. Hawkins RD, Fuller CW. A preliminary assessment of professional footballers' awareness of injury prevention strategies. Br J Sports Med 1998;32:140-143.

12. F-marc. Fifa 11+ - a complete warmup programme. http://f-marc.com (accessed 22 May 2013).

13. Soligard T, Myklebust G, Steffen K, et al. Comprehensive warm-up programme to prevent injuries in female youth football - a cluster randomised controlled trial. BMJ 2008;337:a2469. [http://dx.doi.org/10.1136/bmj.a2469]

14. Twizere J. Epidemiology of soccer injuries in Rwanda: A need for physiotherapy intervention. MSc thesis. Cape Town: University of the Western Cape, 2004. http://etd.uwc.ac.za/usrfiles/ modules/etd/docs/etd_init_1053_1175152032.pdf (accessed 9 July 2013).

15. Steffen K, Emery CA, Romiti M, et al. High adherence to a neuromuscular injury prevention programme (FIFA 11+) improves functional balance and reduces injury risk in Canadian youth female football players: A cluster randomised trial. Br J Sports Med (in press). [http:// dx.doi.org/10.1136/bjsports-2012-091886]

16. Grantham J, Cheung SS, Connes P, et al. Position statement. Current knowledge on playing football in hot environments. Scand J Med Sci Sports 2010;20:161-167.

17. Maughan RJ, Shirreffs SM, Ozgünen KT, et al. Living, training and playing in the heat: Challenges to the football player and strategies for coping with environmental extremes. Scand J Med Sci Sports 2010;20:117-124.

18. Verhagen E, Finch CF. Setting our minds to implementation. Br J Sports Med 2011;45:10151016. [http://dx.doi.org/10.1136/bjsports-2011-090485] 